\title{
Vantagens e desvantagens dos principais tipos de dietas para cães
}

\author{
Advantages and disadvantages of the main types of dog diets \\ Ventajas y desventajas de los principales tipos de dietas para perros
}

Recebido: 18/07/2021 | Revisado: 27/07/2021 | Aceito: 31/07/2021 | Publicado: 06/08/2021

\author{
Bruna Beatriz Voos De Campos \\ ORCID: https://orcid.org/0000-0002-1396-1260 \\ Universidade Estadual de Ponta Grossa, Brasil \\ E-mail: brunabeatrizvc@gmail.com \\ Jessyca Caroline Rocha Ribas \\ ORCID: https://orcid.org/0000-0002-6046-2827 \\ Universidade Estadual de Ponta Grossa, Brasil \\ E-mail: jrocharibas@gmail.com
}

\begin{abstract}
Resumo
O relacionamento entre seres humanos e cães estreitaram-se principalmente nos últimos anos em consequência houve um aumento no setor pet food, disponibilizando uma grande variedade de produtos. Com a alta variedade de alimentos disponíveis no mercado, a escolha do tipo de dieta torna-se um processo complexo e confuso. O objetivo da presente revisão bibliográfica é expor as características das principais formas de alimentação para cães bem como suas vantagens e desvantagens. A maioria dos tutores alimentam seus cães com rações comerciais, pela facilidade de compra e armazenamento. Porém, no processo de fabricação destes alimentos, nem sempre as informações contidas no rótulo condizem com os valores nutricionais do produto e existe inclusão de diversos aditivos químicos. Com a crescente popularidade da alimentação não convencional, as dietas naturais e caseiras oferecem outras possibilidades de escolha dos ingredientes, maior palatabilidade e digestibilidade da dieta, porém, devem ser formuladas adequadamente por profissionais evitando assim carências ou excessos de nutrientes.
\end{abstract}

Palavras-chave: Dietas caseiras; Dietas cruas; Pet food; Rações comerciais.

\begin{abstract}
The relationship between humans and dogs has become closer, mainly in recent years, as a result of an increase in the pet food sector, offering a wide variety of products. With the wide variety of foods available on the market, choosing the type of diet becomes a complex and confusing process. The aim of this literature review is to expose the characteristics of the main forms of feeding for dogs as well as their advantages and disadvantages. Most handlers feed their dogs commercial pet food, for ease of purchase and storage. However, in the manufacturing process of these foods, the information contained on the label does not always match the nutritional values of the product and there is an inclusion of several chemical additives. With the growing popularity of unconventional food, natural and homemade diets offer other possibilities for choosing ingredients, greater palatability and diet digestibility, however, they must be properly formulated by professionals, thus avoiding nutrient deficiencies or excesses.
\end{abstract}

Keywords: Homemade dog food; Raw pet food; Pet food; Wet comercial diets.

\section{Resumen}

La relación entre humanos y perros se ha ido estrechando, principalmente en los últimos años, como consecuencia del aumento del sector de la alimentación para mascotas, que ofrece una amplia variedad de productos. Con la amplia variedad de alimentos disponibles en el mercado, elegir el tipo de dieta se convierte en un proceso complejo y confuso. El objetivo de esta revisión de la literatura es exponer las características de las principales formas de alimentación para perros así como sus ventajas y desventajas. La mayoría de los adiestradores alimentan a sus perros con comida comercial para mascotas, para facilitar la compra y el almacenamiento. Sin embargo, en el proceso de fabricación de estos alimentos, la información contenida en la etiqueta no siempre coincide con los valores nutricionales del producto y hay una inclusión de varios aditivos químicos. Con la creciente popularidad de los alimentos no convencionales, las dietas naturales y caseras ofrecen otras posibilidades de elección de ingredientes, mayor palatabilidad y digestibilidad de la dieta, sin embargo, deben ser adecuadamente formuladas por profesionales, evitando así deficiencias o excesos de nutrientes.

Palabras clave: Dietas caseras; Dietas crudas; Alimentos para mascotas; Comida para perros. 


\section{Introdução}

A crescente proximidade dos animais de companhia com o homem os fazem hoje serem considerados como membros da família (Martens, Enders-Slegers,Walker, 2016; Di Cerbo et al., 2017). Este fato se reflete na tendência dos tutores em escolher os alimentos oferecidos a seus animais da mesma maneira com as quais escolhe a própria alimentação e de seus familiares (Dodd, Adolphe, Verbrugghe, 2018).

Estimativas globais sugerem que mais de $50 \%$ de todas as famílias possuem um gato ou cachorro (Alexander et al., 2020). O Brasil possui a segunda maior população de cães e gatos no mundo, sendo composto por 52 milhões de cães e 22 milhões de gatos (Bragança \& Queiroz, 2021).

Até a década de 80, a maioria dos cães eram alimentados com os restos de comida e poucas indústrias de rações investiam no Brasil (Panjota et al., 2018). Com o aumento do poder aquisitivo, desenvolvimento de pesquisas na área de nutrição animal levando a compreensão das reais necessidades nutricionais de cães e gatos aliados aos investimentos de grandes empresas no setor, esse cenário rapidamente se modificou e atualmente, os alimentos destinados a esses animais buscam além de nutrir, a promoção da saúde e longevidade (Ogoshi et al., 2015; Panjota et al., 2018). O mercado pet food tem tido um crescimento expressivo nos últimos anos abrangendo uma grande diversidade de produtos como rações industrializadas, rações especiais, petiscos, suplementos alimentares e alimentos caseiros (Viana et al., 2020). Em 2019, dentre os serviços pets, o setor pet food representou 73,3\% de todo o faturamento (22,3 bilhões de reais em 2019) com um crescimento de $8,4 \%$ em relação ao ano anterior (ABINPET, 2019).

A escolha do alimento a ser fornecido a cães domiciliados dependem de fatores ligados a seus tutores como suas preferências, nível de informação e condição socioeconômica (Simonsen, Fasenko, Lillywhite, 2014; Boya, Dotson, Hyatt, 2015). A internet e redes sociais tem sido ultimamente um meio comum para busca de informações que irão influenciar na tomada de decisões acerca do alimento a ser fornecido ao animal de companhia (Schleicher, Cash, Freeman, 2019; Viana et al., 2020). Contudo, o excesso de informações e de produtos diferenciados disponíveis no mercado faz com que a escolha da dieta adequada se transforme em um processo confuso (Schleicher, Cash, Freeman, 2019).

Os riscos associados com dietas nutricionalmente inadequadas associam-se ao longo período de fornecimento desta alimentação ao animal, principalmente quando ele ainda não atingiu a idade adulta (Bischoff \& Rumbeiha, 2018). Diante disso, a presente revisão objetiva apresentar as principais dietas disponíveis para alimentação de cães apontando seus pontos positivos e negativos.

\section{Metodologia}

O presente estudo trata-se de uma revisão integrativa da literatura, realizada no primeiro semestre de 2021 sobre as principais dietas fornecidas a cães, ressaltando suas principais características. A revisão abrangeu artigos científicos em língua inglesa e língua portuguesa, através de textos completos publicados entre os anos de 2009 à 2021 e disponíveis nas bases de dados: Capes (Coordenação de Aperfeiçoamento de Pessoal de Nível Superior), Scielo (Scientific Eletronic Library Online) e Google acadêmico. Para realizar a busca das produções científicas foram utilizados os seguintes descritores em inglês: "pet food, natural foods, homemade pet foods, trends in pet food, nutrition of dogs" e em português: alimentos para cães, dietas naturais para cães, dietas caseiras para cães, tendências em pet food, nutrição de cães.

Os artigos encontrados pela estratégia de busca foram primeiramente selecionados pela leitura de seus títulos e resumo. Por último, foram lidos na íntegra, para verificar a adequação ao tema de interesse, visando à seleção final do material de análise. As publicações em teses, dissertações e demais publicações que não atendessem aos objetivos propostos ou que não estavam alinhadas aos critérios de inclusão foram excluídas. 


\section{Resultados e Discussão}

Cães são considerados animais anatomicamente carnívoros por possuírem caninos fortes e bem desenvolvidos, ausência da enzima amilase salivar, estômago pequeno com pH extremamente baixo e intestino grosso curto (Ogoshi et al., 2015). São considerados carnívoros não restritos já que em sua história evolutiva, adquiriram algumas características onívoras (Bragança \& Queiroz, 2021). As exigências nutricionais dos cães devem ser atendidas adequadamente para suprir as demandas energéticas diárias de acordo com seu estado fisiológico, garantindo assim saúde, bem estar e longevidade. A tabela abaixo apresenta as exigências nutricionais de cães em várias fases de vida (fase inicial, crescimento, manutenção e reprodução) e serve como guia para formulação adequada de dietas para cães de diferentes categorias.

Tabela 1: Exigências nutricionais de diferentes categorias.

\begin{tabular}{|c|c|c|c|c|c|}
\hline & & $\begin{array}{c}\text { Inicial } \\
\left(2^{\circ} \text { mês de idade }\right)\end{array}$ & $\begin{array}{c}\text { Crescimento } \\
\text { (do } 3^{\circ} \text { ao } 18^{\circ} \text { mês de } \\
\text { idade) }\end{array}$ & Manutenção & $\begin{array}{r}\text { Reprodução } \\
\text { (Gestação e lactação) }\end{array}$ \\
\hline Energia Metabolizável & $\mathrm{Cal} / \mathrm{Kg}$ & 2.800 & 2.800 & 2.700 & 2.700 \\
\hline Proteína Bruta & $\%$ & 27,0 & 23,0 & 19,0 & 23,0 \\
\hline Fibra Bruta (máxima) & $\%$ & 6,0 & 6,0 & 6,0 & 6,0 \\
\hline Extrato Etéreo & $\%$ & 5,0 & 5,0 & 5,0 & 5,0 \\
\hline Cloreto de Sódio & $\%$ & 1,0 & 1,0 & 1,0 & 1,0 \\
\hline Cálcio & $\%$ & 1,4 & 1,2 & 0,9 & 1,4 \\
\hline Fósforo & $\%$ & 0,8 & 0,7 & 0,6 & 0,6 \\
\hline Potássio & $\%$ & 0,7 & 0,7 & 0,6 & 0,6 \\
\hline Magnésio & $\mathrm{mg} / \mathrm{kg}$ & 900 & 900 & 900 & 900 \\
\hline Ferro & $\mathrm{mg} / \mathrm{kg}$ & 70 & 70 & 50 & 60 \\
\hline Cobre & $\mathrm{mg} / \mathrm{kg}$ & 7 & 7 & 7 & 7 \\
\hline Manganês & $\mathrm{mg} / \mathrm{kg}$ & 50 & 40 & 35 & 35 \\
\hline Cobalto & $\mathrm{mg} / \mathrm{kg}$ & 2 & 2 & 2 & 2 \\
\hline Zinco & $\mathrm{mg} / \mathrm{kg}$ & 30 & 20 & 15 & 15 \\
\hline Iodo & $\mathrm{mg} / \mathrm{kg}$ & 2 & 2 & 2 & 2 \\
\hline Selênio & $\mathrm{mg} / \mathrm{kg}$ & 0,1 & 0,1 & 0,1 & 0,1 \\
\hline Vitamina A & $\mathrm{UI} / \mathrm{kg}$ & 10.000 & 9.000 & 8.000 & 10.000 \\
\hline Vitamina D3 & $\mathrm{UI} / \mathrm{kg}$ & 1.000 & 1.000 & 1.000 & 1.000 \\
\hline Vitamina $\mathrm{E}$ & $\mathrm{mg} / \mathrm{kg}$ & 50 & 50 & 40 & 70 \\
\hline Vitamina K & $\mathrm{mg} / \mathrm{kg}$ & 3 & 3 & 2 & 2 \\
\hline Vitamina $\mathrm{C}$ & $\mathrm{mg} / \mathrm{kg}$ & - & - & - & - \\
\hline Tiamina & $\mathrm{mg} / \mathrm{kg}$ & 3 & 3 & 3 & 3 \\
\hline Riboflavina & $\mathrm{mg} / \mathrm{kg}$ & 5 & 5 & 5 & 5 \\
\hline Piridoxina & $\mathrm{mg} / \mathrm{kg}$ & 3 & 3 & 2 & 3 \\
\hline Vitamina B12 & $\mathrm{mg} / \mathrm{kg}$ & 0,03 & 0,03 & 0,02 & 0,03 \\
\hline Ácido Nicotínico & $\mathrm{mg} / \mathrm{kg}$ & 80 & 70 & 70 & 70 \\
\hline Ácido Pantotênico & $\mathrm{mg} / \mathrm{kg}$ & 30 & 25 & 20 & 35 \\
\hline Ácido Fólico & $\mathrm{mg} / \mathrm{kg}$ & 0,10 & 0,10 & 0,05 & 0,18 \\
\hline Biotina & $\mathrm{mg} / \mathrm{kg}$ & 0,30 & 0,20 & 0,10 & 0,30 \\
\hline Colina & $\mathrm{mg} / \mathrm{kg}$ & 1.200 & 1.100 & 900 & 1.100 \\
\hline
\end{tabular}

Fonte: Valverde (2004).

\section{Tipos de dietas para cães}

Segundo o Ministério da Agricultura, Pecuária e Abastecimento (MAPA) o qual regulamenta a alimentação de cães no Brasil, os alimentos para cães classificam-se com base no seu conteúdo de nutrientes em: completos, coadjuvantes e 
específicos. Alimentos completos são aqueles compostos por ingredientes ou matérias-primas e aditivos destinado exclusivamente à alimentação de animais de companhia que atendem integralmente as exigências animais, podendo possuir propriedades específicas ou funcionais. Os alimentos coadjuvantes são compostos por ingredientes ou matérias-primas e aditivos destinado exclusivamente à alimentação de animais de companhia para animais com distúrbios fisiológicos ou metabólicos, capaz de atender integralmente suas exigências nutricionais específicas, cuja formulação inclui algum agente farmacológico ativo e alimentos específicos são usados para agrado, prêmio ou recompensa e não se caracterizam como alimentos completos (MAPA, 2009). De acordo com o teor de umidade, alimentos destinados aos cães podem ser classificados como: úmido (possuem de 72 a 85\% de água), semi-úmido (possuem de 15 a 50\% de água) ou seco (possuem de 5 a $12 \%$ de água) (MAPA, 2009).

A seguir serão apresentados em tópicos os tipos mais comuns de dietas oferecidas atualmente para cães, apresentando suas principais características, vantagens e desvantagens.

\section{1- Rações convencionais}

As rações comerciais classificam-se de acordo com as indústrias nos tipos: linha econômica, premium e superpremium conforme a matéria-prima utilizada na fabricação (Carciofi et al., 2009; Teixeira \& Ribeiro, 2017). Rações classificadas como econômicas são aquelas fabricadas através de matérias-primas de baixo valor nutricional, sendo elaboradas com subprodutos animais (penas, vísceras, cabeça, osso, entre outros), onde diversos destes ingredientes não são digestíveis aos cães. Geralmente possuem baixa palatabilidade pelo baixo teor de gordura e alta quantidade de ingredientes com baixa digestibilidade podendo ser recusada pelo animal. Alimentos premium apresentam uma seleção minuciosa dos ingredientes, com qualidade nutricional e palatabilidade superior. Produtos super-premium além das características encontradas em rações premium apresentam maior densidade nutricional, ótima digestibilidade, palatabilidade e ingredientes funcionais (Carciofi, 2009).

Os alimentos secos representam a principal forma de alimentar os cães atualmente sendo constituídos por rações extrusadas, apresentando em sua composição ingredientes de origem animal, vegetal, minerais e vitaminas. São de fácil aquisição, possuem custo acessível e podem ser armazenadas por um período prolongado devido seu baixo teor de umidade e a presença de compostos antioxidantes, acidificantes e antifúngicos (Bragança \& Queiroz, 2021).

Apesar das vantagens apresentadas acima, observa-se que, em todas as classificações de rações, os níveis de garantia nem sempre estão de acordo com os dados obtidos após análises laboratoriais (Provenzano et al., 2020) especialmente quando estas rações são elaboradas a partir de subprodutos de origem animal (Wolfarth, Johann \& Araldi, 2011). Teixeira \& Ribeiro (2017) avaliaram doze tipos de rações secas para cães adultos de diferentes classificações. Após análises laboratoriais, constataram que todas as rações apresentaram teores de matéria seca acima daquele apresentado no rótulo. Nas rações classificadas como super-premium, os níveis de proteína se apresentaram mais elevados e extrato etéreo em níveis abaixo do descrito no rótulo. Além disso, todas as rações avaliadas apresentaram excesso de cálcio.

As rações comerciais geralmente são acrescidas de aditivos químicos os quais mantém as características desejáveis do alimento como cor, sabor, textura, estabilidade e resistência à deterioração (Honorato et al., 2013; Craig, 2021). Apesar do aporte legal da utilização de tais aditivos, muitos tutores e pesquisadores consideram que estes possam causar efeitos nocivos à saúde animal, embora há poucos estudos disponíveis para substanciar ou refutar essas suspeitas (Craig, 2019; Craig, 2021).

Sendo assim, todos estes pontos negativos associados ao uso de rações secas comerciais fizeram com que outro segmento do mercado pet food esteja hoje em ascensão: a utilização de dietas não convencionais para animais de companhia, caracterizadas principalmente pela utilização de dietas naturais e caseiras. 


\section{2- Dietas não convencionais}

Hoje em dia, tem-se observado o crescente número de dietas não convencionais destinadas a animais de companhia. Estas podem ser definidas de forma ampla e englobam dietas "naturais" comercialmente disponíveis, dietas vegetarianas e alimentos crus, dietas grain free, bem como, uma variedade de alimentos preparados em casa (dietas caseiras) (Macedo et al., 2018).

O maior interesse dos tutores na busca de dietas não convencionais é pautado na visão de que alimentos mais naturais são mais nutritivos (Macedo et al., 2018; Schleicher, Cash, Freeman, 2019). Além disso, a utilização de dietas não convencionais quando adequadamente balanceadas se faz benéfica, principalmente em animais com dificuldades de consumo, com alergias a componentes utilizados em rações comerciais, animais com problemas de obesidade, renais, endócrinos ou animais oncológicos (Vendramini et al., 2020).

\section{1- Dieta natural}

Hoje, a maioria dos cães ingerem alimentos altamente processados que apresentam pouca semelhança com as dietas ancestrais caninas (Craig, 2021). Em busca de fornecer alimentos menos modificados por industrialização a seus cães, tutores estão recorrendo a dietas naturais. A designação "natural”, abrange os alimentos sem produtos químicos e sem conservantes artificiais e pode ser interpretado pelos tutores como algo mais saudável em relação as demais opções de produtos disponíveis no mercado pet food (Saad \& França, 2010).

De acordo com a AAFCO (Association of American Feed Control Officials) um alimento natural é aquele de origem animal ou vegetal que sofreu ou não ação por agentes físicos (por exemplo: processamento térmico, purificação, extração, hidrólise, fermentação, entre outros) mas que não tenha sido produzido por um processo quimicamente sintético e não contenha quaisquer aditivos químicos sintéticos, exceto em quantidades que possam ocorrer inevitavelmente dentro das boas práticas de fabricação. Neste sentido, a adição de vitaminas e minerais sintéticos somente são permitidos se eles são essenciais a fim de formar um alimento completo e balanceado adequadamente de acordo com as exigências do animal (Carter et al., 2014).

As dietas cruas são consideradas como naturais e baseiam-se no fornecimento de ingredientes não cozidos como carne de músculo crua, carnes de órgãos, gordura, cartilagem e ossos juntamente com vegetais crus (Anturaniemi et al., 2019). Essas dietas podem ser preparadas em casa ou adquiridas frescas, congeladas ou liofilizadas (Davies, Lawes, Wales, 2019).

A crescente popularidade das dietas cruas deve-se aos benefícios defendidos por seus adeptos como: melhora na saúde bucal, pele, pelagem, digestibilidade, qualidade das fezes e melhorias comportamentais (Fredriksson-Ahomaa et al., 2017; Davies, Lawes, Wales, 2019). O processamento térmico pode influenciar negativamente na digestibilidade de um alimento, fato comprovado por Neshovska e Shindarska (2021) onde relatam maior digestibilidade de uma alimentação baseada em alimentos crus para cães em relação a alimentos secos extrusados.

Sandri et al. (2017) compararam os efeitos de uma dieta à base de alimentos crus e com alimentos extrusados na microbiota fecal de cães saudáveis e verificaram que a administração de dietas cruas promoveu um crescimento mais equilibrado das comunidades bacterianas e alteração nos produtos finais de fermentação, com maior concentração de ácido láctico nas fezes dos animais que receberam dieta crua em comparação com extrusada. Em relação a melhoria da saúde bucal, o consumo de ossos crus é eficaz devido ao atrito causado pelo osso em contato com as placas bacterianas nos dentes, reduzindo casos de doença periodontal (Davies, Lawes, Wales, 2019).

O grande risco da utilização deste tipo de dieta refere-se à manipulação de carne crua e os órgãos internos durante o preparo, processamento ou transporte do alimento gerando riscos à saúde humana e animal especialmente indivíduos imunodeprimidos (Morelli et al., 2019). Alguns patógenos zoonóticos presentes nos alimentos crus para animais de estimação 
incluem Escherichia coli, espécies de Salmonella, espécies de Clostridium, Listeria monocytogenes, espécies de Yersinia e Campylobacter (Fredriksson-Ahomaaet al., 2017; Anturaniemi et al., 2019).

Nilsson (2015) avaliou 39 amostras de oito alimentos crus para cães de diferentes marcas e confirmou a presença da bactéria Escherichia coli em todas as amostras. Hellgren et al. (2019) avaliaram 60 amostras congeladas de dietas à base de carne crua (RMBDs) para cães produzidas por dez fabricantes diferentes e identificaram a presença de Enterobacteriaceae em todas as amostras, Salmonella em quatro amostras e espécies de Campylobacter em três amostras. Estes dados sugerem que é fundamental manter uma boa higiene durante o preparo, armazenamento e manuseio de alimentos crus, principalmente carnes e subprodutos cárneos, a fim de limitar os riscos potenciais à saúde de animais e humanos.

Morelli et al. (2019) entrevistaram 218 tutores de cães onde 94\% consideram dietas RMBDs segura para seus animais de estimação e relatam que seus animais tiveram pelagem mais brilhante, ganho de massa muscular e dentes mais limpos. 57\% dos entrevistados consideram como principais vantagens o controle da composição e qualidade dos ingredientes fornecidos aos seus animais e como principais desvantagens a compra de alguns ingredientes (38\%) e ao tempo gasto na preparação da dieta (22\%). Na mesma entrevista, somente $8 \%$ dos entrevistados confiaram em veterinários para a formulação de RMBD, o que pode ser perigoso visto que desbalanços nutricionais em dietas fornecidas por um período prolongado ao animal podem resultar em consequências severas a saúde dos mesmos, principalmente em animais jovens.

Hall et al. (2020) apresenta o caso de um Bulldog de sete meses de idade que era alimentado com dieta crua e apresentava sintomas clínicos de letargia progressiva, intolerância ao exercício, paresia dos membros posteriores, atrofia muscular e hiperestesia. O paciente foi diagnosticado com osteopenia acentuada com hipovitaminose $\mathrm{D}$ grave, hipocalcemia $\mathrm{e}$ hiperparatiroidismo, hipovitaminose A, hipotireoidismo e pneumonia grave concomitante os quais estavam associados aos desbalanços nutricionais da dieta fornecida.

\section{2- Dietas caseiras}

O desejo de cozinhar para seu animal, utilizando ingredientes frescos, orgânicos, sem aditivos químicos, a ideia de fornecer uma dieta variada todos os dias são apenas alguns dos motivos pelos quais as dietas caseiras estão ganhando cada vez mais popularidade (Remillard \& Crane, 2010). Este tipo de dieta possui os benefícios de fornecer melhor digestibilidade, palatabilidade e qualidade das fezes quando comparada como dietas comerciais. Destaca-se também que as dietas caseiras podem ser uma ferramenta útil para o manejo nutricional de cães com certas doenças (Oliveira et al., 2014)

Felix et al. (2009) realizaram um estudo com o objetivo de comparar os coeficientes de digestibilidade aparente (CDA) e a qualidade das fezes de seis cães alimentados com dieta caseira e duas rações comerciais: uma classificada como econômica e a outra como superpremium. Como resultados, a dieta caseira apresentou os maiores CDA, e a ração econômica apresentou menor digestibilidade, gerando o maior resíduo fecal. Conceição, Goslar e Silva (2016) ao fornecer dieta caseira para cão obeso observaram alta digestibilidade, reduzida quantidade de fezes, as quais se caracterizaram como pouco ácidas, secas e de consistência firme.

Em geral, as dietas caseiras exigem um preparo mais minucioso (Stockman et al., 2013), custo maior (Macedo et al., 2018; Vendramini et al., 2020) e possuem tempo de conservação curto. Ainda, deve-se prestar atenção a alimentos que possuem componentes tóxicos.

Ressalta-se que somente um profissional especializado em nutrição de cães será capaz de formular adequadamente uma dieta não convencional ao animal, conforme suas necessidades. Além disso, deve fornecer instruções bem claras em relação ao preparo da dieta sem a possibilidade do tutor de modificar a fórmula recomendada pelo profissional. Modificações de ingredientes, qualidade e proporção podem alterar a composição nutricional da dieta, gerando desequilíbrios nutricionais 
(Remillard \& Crane, 2010; Oliveira et al., 2014). Tal et al. (2018) relataram deficiência de vitamina D e hiperparatireoidismo nutricional secundário em um cão com seis meses de idade alimentado com uma dieta caseira nutricionalmente desequilibrada.

Halfen et al. (2017) ao entrevistarem 55 proprietários que forneciam dieta caseira para seus cães na cidade de São Paulo concluíram que $60 \%$ destes admitiram modificar as formulações prescritas. Oliveira et al. (2014) em entrevista com 46 tutores que utilizam dietas caseiras prescritas por veterinários relatam que destes, 30,4\% admitiram modificar as dietas, $40 \%$ não controlaram adequadamente a quantidade de ingredientes fornecidos, 73,9\% não usaram as quantidades recomendadas de óleo de soja e sal e 34,8\% não usaram corretamente os suplementos vitamínicos e minerais.

Assim, apesar dos benefícios da utilização de dietas não convencionais aos cães, essas dietas devem ser formuladas por profissionais capacitados. Cuidados devem ser tomados em relação a escolha dos ingredientes, formulação adequada para atender as exigências nutricionais do animal, orientação correta aos tutores a respeito da administração deste tipo de alimento e check-ups de rotina para avaliar a saúde destes animais.

\section{Considerações Finais}

Diversos tipos de dietas estão disponíveis no mercado para os cães e a escolha deve ser pautada considerando fatores relacionados ao animal e as características de cada dieta. As rações constituem a principal forma de alimentar os cães, devido a sua praticidade e acessibilidade. Suas características diferem quanto as linhas econômica, premium e superpremium, podendo ser fabricadas a partir de subprodutos de origem animal, até por ingredientes funcionais. Porém os níveis de garantia descritos no rótulo nem sempre conferem com os dados obtidos após análises laboratoriais. Além disso, o uso de corantes e outros aditivos químicos podem causar reações de hipersensibilidade aos animais.

A popularidade das dietas não convencionais vem crescendo, sendo representadas por dietas naturais, caseiras, entre outras. Geralmente possuem maior seleção de ingredientes, palatabilidade e digestibilidade, porém, este tipo de alimentação necessita de cuidados relacionados a contaminação microbiana e relacionados aos desbalanços de nutrientes quando a formulação não atende as exigências nutricionais do animal.

\section{Referências}

ABINPET. (2019). "Mercado Pet Brasil". Website da Asssociação Brasileira da Indústria de Produtos para Animais de Estimação (ABINPET). http://abinpet.org.br"

Alexander, P., Berri, A., Moran, D., Reay, D. \& Rounsevell, M. (2020). The global environmental paw print of pet food. Global Environmental Change, 65, 102153.

Anturaniemi, J., Barrouin-Melo, S. M., Zaldivar-López, S., Sinkko, H. \& Hielm-Björkman, A. (2019). Owners' perception of acquiring infections through raw pet food: a comprehensive internet-based survey. Vet Rec, 30;185(21):658.

Association of American Feed Control Officials. Feed terms and ingredient definitions. In: Association of American Feed Control Officials official publication. Oxford, Ind: Association of American Feed Control Officials, 2013;336-460.

Bischoff, K., Rumbeiha, W. K. (2018). Pet Food Recalls and Pet Food Contaminants in Small Animals: An Update. Vet Clin North Am Small Anim Pract. 48(6):917-931.

Boya, U. O., Dotson, M. J. \& Hyatt, E. M. (2015). A comparison of dog food choice criteria across dog owner segments: An exploratory study. Int J Consum Stud 39, 74-82.

Bragança, D. F. \& Queiroz, E. O. (2021). Manejo nutricional de cães e gatos e as tendências no mercado pet food: Revisão. PUBVET, 15(02), 1-11.

Carter, R. A., Bauer, J. E., Kersey, J. H. \& Buff, P. R. (2014). Awareness and evaluation of natural pet food products in the United States. Vet Med Today: Timely Topics in Nutrition, 245(11):1241-8.

Carciofi, A. C., Teshima, E., Bazolli, R. S. et al. (2009). Qualidade e digestibilidade de alimentos comerciais de diferentes segmentos de mercado para cães adultos. Rev. Bras. Saúde Prod. An., 10(2), 489-500.

Craig, J. M. (2019). Food intolerance in dogs and cats. Journal of Small Animal Practice, 60, 77-85. 
Craig, J. M. (2021). Additives in pet food: are they safe? Journal of Small Animal Practice, 1-12.

Conceição, O. S., Goslar, M. S. \& Silva, A. L. P. (2016) Avaliação da qualidade de dietas caseiras para cães obesos. Revista Eletrônica Biociências, Biotecnologia e Saúde, Curitiba, 15.

Davies, R. H, Lawes, J. R. \& Wales, A. D. (2019). Raw diets for dogs and cats: a review, with particular reference to microbiological hazards. J Small Anim Pract., 60(6):329-339.

Di Cerbo, A., Morales-Medina, J. C., Palmieri, B., Pezzuto, F., Cocco, R.; Flores, G.; Iannitti, T. (2017). Functional foods in pet nutrition: Focus on dogs and cats. Research in Veterinary Science, 112, 161-166.

Dodd, S. \& Adolphe, J. \& Verbrugghe, A. (2018). Plant-based diets for dogs. Journal of the American Veterinary Medical Association. 253. $1425-1432$.

Felix, A. P., Sá-Fortes, C. M. L., Silva, A. C. M. et al. (2009). Digestibilidade de uma dieta caseira e dois alimentos comerciais, econômico e super-prêmio para cães. Archives of Veterinary Science, 14(1), 25-30.

Fredriksson-Ahomaa, M., Heikkilä, T., Pernu, N., Kovanen, S., Hielm-Björkman, A. \& Kivistö, R. (2017). Raw Meat-Based Diets in Dogs and Cats. Veterinary sciences, 4(3), 33.

Halfen, D. P., Oba, P. M., Duarte, C. N. et al. (2017). Tutores de cães consideram a dieta caseira como adequada, mas alteram as fórmulas prescritas. Pesq. Vet. Bras., 37(12), 1453-1459, 2017.

Hall, G., Breheny, C., Khan, Z., Shwarz, T. \& Mellanby, R. J. (2020). Severe nutritional deficiencies and osteopenia in a dog fed a homemade raw diet. Vet Report Case Reports, 8. e001038.

Honorato, T. C., Batista, E., Nascimento, K. O. \& Pires, T. (2013). Aditivos alimentares: aplicações e toxicologia. Revista Verde, 8(5), 1-11.

Macedo, H. T., Pedrinelli, V., Fragoso, R. M. et al. (2018). Alimentos não convencionais para cães e gatos. In: Novos desafios da pesquisa em nutrição e produção animal. 90-95.

MAPA. Instrução Normativa $n^{\circ} 30$ de 2009. Sistema de Consulta a legislação. http://sistemasweb.agricultura.gov.br.

Martens, P., Enders-Slegers, M. \& Walker, J. K. (2016) The Emotional Lives of Companion Animals: Attachment and Subjective Claims by Owners of Cats and Dogs, Anthrozoös, 29(1), 73-88.

Morelli, G., Bastianello, S., Catellani, P. et al. (2019). Raw meat-based diets for dogs: survey of owners' motivations, attitudes and practices. BMC Vet $\operatorname{Res} 15,74$.

Neshovska, H. \& Shindarska, Z. (2021). Comparative study of the digestibility of dry and raw food in dogs. International Journal of Veterinary Sciences and Animal Husbandry, 6(2), 1-3.

Nilsson, O. (2015). Hygiene quality and presence of ESBL-producing Escherichia coli in raw food diets for dogs. Infection ecology \& epidemiology, 5 , 28758.

Ogoshi, R. C. S., Reis, J. S., Zangeronimo, M. G. \& Saad, F. M. O. (2015). Conceitos básicos sobre nutrição e alimentação de cães e gatos. Ciência Animal, $25(1) ; 64-75$.

Oliveira, M., Brunetto, M., Da Silva, F. et al. (2014). Evaluation of the owner's perception in the use of homemade diets for the nutritional management of dogs. Journal of Nutritional Science, 3, E23.

Pantoja, J. C., Cabral, Í. S., Farias, T. S., Amaral, T. E. S. \& Barbosa, C. R. (2018). Alimentação de cães e gatos cardiopatas. PUBVET, 12(11), 1-8.

Provenzano, A. C. G., Diana, T. F., Marques, K. C., Teixeira, A. O. \& Reis, R. S. (2020). Qualidade nutricional de rações secas de diferentes segmentos comerciais para cães. Research, Society and Development, 9(11), e1469119590.

Remillard, R. L. \& Crane, S. W. (2010). Chapter 10-Making Pet Foods at Home. In: Small Animal Clinical Nutrition, 5th Edition.

Saad, F. M. O. B. \& França, J. (2010). Alimentação natural para cães e gatos. R. Bras. Zootec., 39, 52-59.

Sandri, M., Dal Monego, S., Conte, G. et al. (2017). Raw meat based diet influences faecal microbiome and end products of fermentation in healthy dogs. BMC Vet Res, 13(65).

Schleicher, M., Cash, S. B \& Freeman, L. M. (2019). Determinants of pet food purchasing decisions. The Canadian veterinary journal = La revue veterinaire canadienne, 60(6), 644-650.

Simonsen, J. E., Fasenko, G. M., Lillywhite, J. M. (2014). The value-added dog food market: Do dog owners prefer natural or organic dog foods? J Ag Sci 6, 86-97.

Stockman, J., Fascetti, A. J., Kass, P. H. \& Larsen, J. A. (2013). Evaluation of recipes of home-prepared maintenance diets for dogs. Javma, 242(11).

Tal, M., Parr, J. M., MacKenzie, S. \& Verbrugghe, A. (2018). Dietary imbalances in a large breed puppy, leading to compression fractures, vitamin D deficiency, and suspected nutritional secondary hyperparathyroidism. The Canadian veterinary journal = La revue veterinaire canadienne, 59(1), 36-42.

Teixeira, J. B. D. \& Ribeiro, M. (2017). Avaliação bromatológica das principais rações secas de cães. Saber Digital, 10(2), 105-115.

Valverde, C. C. (2004). 250 Maneiras de Preparar Rações Balanceadas para Cães. Viçosa - MG. Viçosa: Aprenda Fácil. 110. 
Research, Society and Development, v. 10, n. 10, e91101018368, 2021

(CC BY 4.0) | ISSN 2525-3409 | DOI: http://dx.doi.org/10.33448/rsd-v10i10.18368

Vendramini, T. H. A., Pedrinelli, V., Macedo, H.T. et al. (2020). Homemade versus extruded and wet commercial diets for dogs: Cost comparison. PLoS One. 24;15(7):e 0236672.

Viana, L. M., Mothé, C. G. \& Mothé, M. G. (2020). Natural food for domestic animals: A national and international technological review. Research in Veterinary Science, 130, 11-18.

Wolfarth, D., Johann, M. \& Araldi, D. (2011). A importância de uma dieta de qualidade na alimentação de cães e gatos. XVI seminário interinstitucional de ensino, pesquisa e extensão. UNICRUZ. 